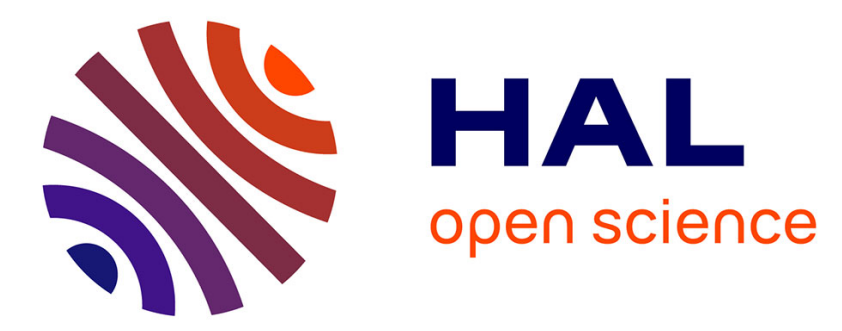

\title{
Quantised control of nonlinear systems: analysis of robustness to parameter uncertainty, measurement errors, and exogenous disturbances
}

Alessio Franci, Antoine Chaillet

\section{- To cite this version:}

Alessio Franci, Antoine Chaillet. Quantised control of nonlinear systems: analysis of robustness to parameter uncertainty, measurement errors, and exogenous disturbances. International Journal of Control, 2010, 83 (12), pp.2453-2462. 10.1080/00207179.2010.529464 . hal-00546723

HAL Id: hal-00546723

https://hal-centralesupelec.archives-ouvertes.fr/hal-00546723

Submitted on 14 Dec 2010

HAL is a multi-disciplinary open access archive for the deposit and dissemination of scientific research documents, whether they are published or not. The documents may come from teaching and research institutions in France or abroad, or from public or private research centers.
L'archive ouverte pluridisciplinaire HAL, est destinée au dépôt et à la diffusion de documents scientifiques de niveau recherche, publiés ou non, émanant des établissements d'enseignement et de recherche français ou étrangers, des laboratoires publics ou privés. 


\title{
RESEARCH ARTICLE
}

\section{Quantized Control of Nonlinear Systems: a Robust Approach}

\author{
Alessio Franci ${ }^{a *}$ and Antoine Chaillet $^{b}$ \\ ${ }^{a}$ LSS - Supélec - Université Paris Sud, 3 rue Joliot-Curie, 91192 Gif sur Yvette, France; ${ }^{b}$ EECI - LSS - \\ Supélec - Université Paris Sud, 3 rue Joliot-Curie, 91192 Gif sur Yvette, France \\ (Received 00 Month 200x; final version received 00 Month 200x)
}

\begin{abstract}
We propose a variant of the recently introduced strategy for stabilization with limited information [D. Liberzon and J. P. Hespanha. "Stabilization of nonlinear systems with limited information feedback." IEEE Trans. on Automat. Contr., 2005] and analyze its robustness properties. We show that, if the nominal plant can be made Input-to-State Stable (ISS) with respect to measurement errors, parameter uncertainty and exogenous disturbances, then this robustness is preserved with this quantized feedback. More precisely, if a sufficient bandwidth is available on the communication network, then the resulting closed-loop is shown to be semiglobally Input-to-State practically Stable (ISpS).
\end{abstract}

Keywords: limited-information feedback; robustness; nonlinear systems

\section{Introduction}

The always greater use of digital communication devices for control applications makes quantization a crucial issue. The limitations on the communication rate between the plant sensors and its controller imposes to develop new approaches that are able to guarantee good performance even when only limited information on the plant's state is available. Despite strong technological improvements, the bit rate available for a given control application may indeed be strongly limited due to scalability or energy-saving concerns, or due to harsh environment constraints. Stabilization in this context becomes particularly challenging in presence of model uncertainties, measurement errors or exogenous disturbances.

These observations explain why limited-information control feedback has been widely studied recently (see Nair et al. (2007), Hespanha et al. (2007), Liberzon (2008) and references therein for representative examples). An important literature already exists for linear systems (cf. e. g. Montestruque and Antsaklis (2004), Liberzon (2003), Petersen and Savkin (2001), Nair and Evans (2004), Jaglin et al. (2008, 2009)). In particular, the results of Liberzon and Nešić (2007) provide a coding/decoding strategy that achieves Input-to-State Stabilization of quantized linear control systems. The proposed control strategy relies on a discrete time zoom-in/zoom-out procedure. This construction is based on the exact sampled dynamics of the system, or at most on its discrete time approximation. This is why the closed loop system may lack robustness with respect to parameter uncertainties. These results were subsequently generalized to nonlinear systems in Kameneva and Nešić (2008). It is then of interest to analyze the robustness of different approaches to guarantee the same performance in the presence of model uncertainties.

In Sharon and Liberzon (2007), Input-to-State Stabilization of quantized linear and nonlinear systems is achieved in the framework of continuous time quantized control systems, that is

*Corresponding author. Email: alessio.franc@lss.supelec.fr 
exploiting a hybrid dynamics. It is based on a generalization of the well known dynamic quantization approach developed in Liberzon and Hespanha (2005) and Persis and Isidori (2004) for ISS and global asymptotically stable systems respectively. For nonlinear systems this control strategy leads to local ISS. The stability region and the size of tollerated perturbations can be eventually enlarged by allowing larger overshoots of the transient dynamics, although this may not hold true in general (see Section 5). Moreover, this approach is not robust to parametric uncertainties as detailed in Section 5.

The purpose of this paper is then to propose an alternative dynamic quantization strategy, able to cope with (time varying) model uncertainties. It is based on a simple and natural modification of the one proposed in Liberzon and Hespanha (2005). We show that the controlled system is semiglobally Input-to-State practical Stable. The semiglobal characterization of our results implies that for any compact set of initial conditions and for any bounded time varying measurement error, disturbance and model uncertainty, it is possible to achieve the desired robustness properties by properly tuning the controller parameters. On the other hand, practical stability does not guarantee convergence to the origin for vanishing perturbations, although the size of the stable subset depends on the tuning parameters and can be somewhat reduced, provided a sufficient knowledge on the intensity of the perturbations. The main contributions of our work are the robustness to model uncertainties and its semiglobal characterization for nonlinear systems. At the light of the poorer performances of the proposed strategy in terms of the granted steady-state error, one may think of implementing some switching strategy involving both the methods methods.

The rest of the paper is organized as follows. In Section 2 we introduce the needed notation. In Section 3 we formally state the problem. In Section 4 we introduce our dynamic quantization strategy. We then present the main results of the paper and comment them in Section 5. In Section 6 we check their application with numerical simulations on the illustrative example of a DC motor with nonlinear load. Proofs are given in Section 7.

\section{Notation}

For a set $A \subset \mathbb{R}$, and $a \in A, A_{\geq a}$ denotes the set $\{x \in A: x \geq a\} .|x|$ denotes the infinity norm of the vector $x$, that is, if $x \in \mathbb{R}^{n},|x|:=\max _{i=1, \ldots, n}\left|x_{i}\right| . B(x, R)$ refers to the closed ball of radius $R$ centered at $x$ in this norm, i.e. $B(x, R):=\left\{z \in \mathbb{R}^{n}:|x-z| \leq R\right\} .\|x\|$ is the infinity norm of the signal $x(\cdot)$, that is, if $x: \mathbb{R}_{\geq 0} \rightarrow \mathbb{R}^{n},\|x\|=\operatorname{esssup}_{t \geq 0} \max _{i=1, \ldots, n}\left|x_{i}(t)\right|$. A continuous function $\alpha: \mathbb{R}_{\geq 0} \rightarrow \mathbb{R}_{\geq 0}$ is said to be of class $\mathcal{K}$ if it is increasing and $\alpha(0)=0$. It is said to be of class $\mathcal{K}_{\infty}$ if it is of class $\mathcal{K}$ and $\alpha(s) \rightarrow \infty$ as $s \rightarrow \infty$. A function $\beta: \mathbb{R}_{\geq 0} \times \mathbb{R}_{\geq 0} \rightarrow \mathbb{R}_{\geq 0}$ is said to be of class $\mathcal{K} \mathcal{L}$ if $\beta(\cdot, t) \in \mathcal{K}$ for any fixed $t \geq 0$ and $\beta(s, \cdot)$ is decreasing and tends to zero at infinity for any fixed $s \geq 0$.

\section{Problem statement}

We are interested in the robustness properties of nonlinear plants of the form

$$
\dot{x}=f(x, \mu, u, d),
$$

where $x \in \mathbb{R}^{n}$ is the state, $f: \mathbb{R}^{n} \times \mathbb{R}^{p} \times \mathbb{R}^{m} \times \mathbb{R}^{h} \rightarrow \mathbb{R}^{n}$ is a locally Lipschitz function, $\mu: \mathbb{R}_{\geq 0} \rightarrow \mathcal{P} \subset \mathbb{R}^{p}$ is a vector of (possibly time-varying) parameters, $u: \mathbb{R}_{\geq 0} \rightarrow \mathbb{R}^{m}$ is a control input and $d: \mathbb{R}_{\geq 0} \rightarrow \mathcal{D} \subset \mathbb{R}^{h}$ is a vector of measurable and locally essentially bounded exogenous perturbations. We assume that $f(0, \mu, 0,0)=0$ for all $\mu \in \mathcal{P}$.

As we are interested in limited-information feedback, we assume that only an estimate of the state is available to the controller. This estimate is elaborated based on an encoded measurement of the actual state. This encoded symbol is then sent over the communication channel. We 
Figure 1. Structure of the quantized control system

will assume, in this paper, that the communication channel is noiseless and delay-free. The communication channel is defined by its constant sampling period $\tau$ and by the number of symbols that can be transmitted at each sampling time $N^{n}, N \in \mathbb{N}_{>0}$. The overall structure of the controlled systems can be summarized by the following figure.

At each reception of a symbol, that is, at each time instant $k \tau, k \in \mathbb{N}$, the decoder computes the state estimate that will be used in the applied feedback law. The decoding is necessarily imprecise due to the limited bandwidth of the channel. This imprecision is reinforced by the uncertainty on the plant parameter $\mu$, by the presence of exogenous disturbances and by the possible measurement errors. We assume that only a constant ${ }^{1}$ approximation $\nu \in \mathcal{P}$ of the (possibly time-varying) parameter vector $\mu$ is available and define $\mu-\nu=: d_{p} \in \mathbb{R}^{p}$ as the parameter uncertainty. We let $d_{e} \in \mathbb{R}^{n}$ denote the measurement error. Our first assumption imposes that, when no communication constraint applies, the plant (1) can be stabilized by a state-feedback law that makes it ISS with respect to exogenous disturbances, parameter uncertainties and measurement errors.

Assumption 3.1 There exists a continuous feedback law $\kappa: \mathbb{R}^{n} \times \mathbb{R}^{p} \rightarrow \mathbb{R}^{n}$, a continuously differentiable function $V: \mathbb{R}^{n} \rightarrow \mathbb{R}$ and class $\mathcal{K}_{\infty}$ functions $\underline{\alpha}, \bar{\alpha}, \alpha, \chi, \Gamma, \gamma$ such that, for all $x \in \mathbb{R}^{n}, d \in \mathcal{D}, d_{p} \in \mathcal{P}$ and $d_{e} \in \mathbb{R}^{n}$,

$$
\underline{\alpha}(|x|) \leq V(x) \leq \bar{\alpha}(|x|),
$$

where $\mathcal{D} \subset \mathbb{R}^{h}$ and $\mathcal{P} \subset \mathbb{R}^{p}$, and the following implication holds true

$$
\begin{gathered}
|x| \geq \chi(|d|)+\Gamma\left(\left|d_{p}\right|\right)+\gamma\left(\left|d_{e}\right|\right) \quad \Rightarrow \\
\frac{\partial V}{\partial x} f\left(x, \mu, \kappa\left(x+d_{e}, \mu+d_{p}\right), d\right) \leq-\alpha(|x|) .
\end{gathered}
$$

Based on the Lyapunov characterization of ISS systems Sontag and Wang (1995), condition (2) is equivalent to ISS of (1) with respect to $d, d_{e}, d_{p}$, at least locally as far as $d$ and $d_{p}$ are concerned. Assumption 3.1 therefore constitutes a strong requirement, but the following remarks may help establishing it in some particular contexts.

Remark 1: For all systems in strict feedback form it is possible to achieve conditions of Assumption 3.1. This comes from the fact that a backstepping procedure can naturally be applied to systems in that form. Such a procedure allows to iteratively make each state element ISS with respect to $\left(d, d_{p}, d_{e}\right)$, using part of the state as a "virtual" control input (see Freeman and Kokotovich (1993) for details).

Remark 2: The conditions of Assumption 3.1 can be achieved for all systems which can be stabilized by a globally Lipschitz state feedback that makes it ISS with respect to actuation errors. Indeed if $\mathcal{L}$ denotes the global Lipschitz constant of the nominal control law $\kappa$, then the effects due to parameter uncertainties $d_{p}$ and measurement errors $d_{e}$ can be described explicitly as an input disturbance $\tilde{d}$ satisfying $|\tilde{d}| \leq \mathcal{L}\left|d_{p}\right|+\mathcal{L}\left|d_{e}\right|$. Hence, if $\gamma \in \mathcal{K}_{\infty}$ is the ISS gain, the presence of measurement errors and parameter uncertainties simply adds $\gamma(|\tilde{d}|) \leq \gamma\left(2 \mathcal{L}\left|d_{p}\right|\right)+\gamma\left(2 \mathcal{L}\left|d_{e}\right|\right)$ to the solution estimate of the closed-loop solutions, hence proving ISS with respect to $\left(d_{p}, d_{e}\right)$. Note that all systems which can be made ISS by differentiable bounded control (cf. e.g. (A.Isidori 1999, Chapters 12,13,14)) trivially satisfy this global Lipschitz condition. $\triangleleft$

\footnotetext{
${ }^{1}$ In a second stage one may think of implementing an adaptive control strategy.
} 


\section{Quantized controller}

In this section, we extend the encoding-decoding procedure presented in Liberzon and Hespanha (2005) and Persis and Isidori (2004) to take into account exogenous disturbances, measurement errors and parameter uncertainties. We assume that measurement errors are bounded by

$$
E>0, \text { such that }\left\|d_{e}\right\| \leq E
$$

\subsection{Quantization region}

Given an estimate $\hat{x}$ of the actual state $x$, the quantization region $\mathcal{Q}$ is defined by its centroid $\hat{x}$ and its radius $L>0$ as

$$
\mathcal{Q}:=B(\hat{x}, L) .
$$

Due to measurement errors, the information available to the encoder about the system state, i.e. $x+d_{e}$, belongs to the quantization region $\mathcal{Q}$ if and only if the estimation error $e:=x+d_{e}-\hat{x}$ is small enough, that is $|e| \leq|x-\hat{x}|+|E| \leq L$. Note that the presence of the estimation error $e$ results from the combined effects of quantization $(x-\hat{x})$ and measurement errors $\left(d_{e}\right)$. Given the number $N^{n}$ of symbols that can be transmitted through the communication channel, we partition the quantization region into $N^{n}$ identical hypercubes. $\mathcal{Q}$ is then updated according to the following encoding-decoding procedure.

\subsection{Dynamics of the encoder}

At each step $k \in \mathbb{N}$, the centroid update law is given by the following hybrid dynamics

$$
\begin{aligned}
\dot{\hat{x}} & =f(\hat{x}, \nu, \kappa(\hat{x}, \nu), 0), \quad \forall t \in[k \tau,(k+1) \tau), \\
\hat{x}(k \tau) & =\hat{c}(k \tau), \quad k \neq 0, \\
\hat{x}\left(0^{-}\right) & =0
\end{aligned}
$$

where $\hat{c}(k \tau)$ is the centroid of the sub-region of $\mathcal{Q}(k \tau)$ in which $x(k \tau)+d_{e}$ lies. This sub-region is identified by the variable $q_{k}$, which constitutes the output of the encoder. In other words, $q_{k} \in \mathbb{N}_{\leq N^{n}}$ denotes the index of the sub-region of $\mathcal{Q}(k \tau)$ to which $x(k \tau)+d_{e}$ belongs. Then, given some $\Lambda>1$ and any ball of initial conditions $B(0, \Delta)$, with $\Delta>0$, the radius update law is given, at each step $k \in \mathbb{N}$, by the following dynamics

$$
\begin{aligned}
L((k+1) \tau) & =\Lambda\left(\frac{L(k \tau)}{N}+E\right)+E, \\
L(0) & =\Delta+E .
\end{aligned}
$$

This radius update law is a natural extension of the algorithms proposed in Liberzon and Hespanha (2005), Persis and Isidori (2004). It takes into account possible measurement errors. We will show in the sequel (cf. Claim 7.2) that such a dynamics leads to a sequence $\{L(k \tau)\}_{k \in \mathbb{N}}$ that decreases up to a constant depending on $E, \Lambda$ and $N$. This in turn imposes a decrease of the estimation error, modulo the measurement errors, as long as dynamics (5) applies. The idea behind this dynamics can be roughly summarized as follows. The parameter $\Lambda>1$ accounts for the expansiveness between sampling times. The constant $E$ appearing inside the round brackets of $(5 \mathrm{a})$ accounts for the case in which the measurement error has let the encoder individuate a wrong sub region. In such a situation the error between the real and the measured state is indeed less than the size of the sub-region, $L(k \tau) / N$, plus the measurement error. The second $E$ 
appearing in (5a) avoids that the measured state falls out of the quantization region while the real one is inside.

Note that, as long as $x(k \tau)+d_{e}$ lies in $\mathcal{Q}(k \tau)$, the estimation error satisfies $|e(k \tau)| \leq \bar{e}(k \tau)$, where

$$
\bar{e}(k \tau):=\frac{L(k \tau)}{N}+E, \quad \forall k \in \mathbb{N},
$$

is the maximum quantization error. Hence, at each sampling time, the quantization procedure individuates a hypercube $B(\hat{x}(k \tau), \bar{e}(k \tau))$ to which $x(k \tau)$ belongs, provided that $x(k \tau)+d_{e} \in$ $\mathcal{Q}(k \tau)$.

However, due to uncertainties and disturbances, it may happen that $x(k \tau)+d_{e}$ falls out of the quantization region anyway. Indeed, the expansion factor $\Lambda$ in (5a) makes sure that the updated quantization region is large enough to catch the measured state only if the quantization error is large compared to the disturbances (see proof of Theorem 5.1 for details). This situation is defined as an overflow. It is represented by the symbol $q_{k}=0$. If an overflow occurs at the $k_{0}$ th sampling time, $k_{0} \in \mathbb{N}_{>0}$, the encoder updates the quantization region as follows:

$$
\begin{aligned}
\hat{x}\left(k_{0} \tau\right) & =\hat{x}\left(k_{0} \tau^{-}\right), \\
L\left(\left(k_{0}+1\right) \tau\right) & =\Lambda(\bar{E}+E)+E,
\end{aligned}
$$

where $\bar{E} \in \mathbb{R}_{>0}$ will be defined later on. This means that the hypercube individuated by the quantization procedure, to which $x(k \tau)$ belongs, is no longer $B\left(\hat{x}(k \tau), \frac{L(k \tau)}{N}+E\right)$, but rather $B(\hat{x}(k \tau), \bar{E}+E)$, while the rest of the update law remains as in (4)-(5).

\subsection{Dynamics of the decoder}

By implementing the same evolution laws as (4)-(7), the decoder is able to reconstruct the evolution of the state estimate $\hat{x}$ from the knowledge of $\left\{q_{k}\right\}_{k \in \mathbb{N}}$.

\subsection{Controller}

Inspired by the principle of certainty equivalence, and in view of Assumption 3.1, the applied control input is given by

$$
\hat{u}(t)=\kappa(\hat{x}(t), \nu)
$$

where $\hat{x}(\cdot)$ is given by (4) along with (5)-(7).

\section{Main Results}

Our first result establishes robustness properties of the closed-loop system with the proposed limited-information feedback in the case the number of transmittable bits is fixed and the sampling period can be adjusted arbitrarily.

Theorem 5.1: $\quad$ Let Assumption 3.1 hold for the system (1). Then, there exist class $\mathcal{K}_{\infty}$ functions $\bar{\chi}, \bar{\Gamma}, \bar{\gamma}$ and, given any compact sets $\mathcal{P} \subset \mathbb{R}^{p}$ and $\mathcal{D} \subset \mathbb{R}^{d}$, any constant $\Delta \in \mathbb{R}_{>0}$ and any $N \in \mathbb{N}_{>1}$, there exist positive constants $\tau, \Lambda, \bar{E}, E$ and a class $\mathcal{K} \mathcal{L}$ function $\beta$ such that the trajectories of the closed-loop system

$$
\dot{x}=f(x, \mu, \hat{u}, d),
$$


where $\hat{u}(t)$ is the output of the digital controller defined by (4)-(8), satisfy, for all $x(0) \in B(0, \Delta)$, all $\nu \in \mathcal{P}$, all $\mu: \mathbb{R}_{\geq 0} \rightarrow \mathcal{P}$, all $d: \mathbb{R}_{\geq 0} \rightarrow \mathcal{D}$ and all $d_{e}: \mathbb{R}_{\geq 0} \rightarrow B(0, E)$,

$$
|x(t)| \leq \bar{\beta}(\Delta, t)+\bar{\chi}(\|d\|)+\bar{\Gamma}(\|\mu-\nu\|)+\delta,
$$

where $\delta=\bar{\gamma}\left(\Lambda(\bar{E}+E)+\left(2+\frac{\Lambda+1}{1-\frac{\Lambda}{N}}\right) E\right)$.

That is, (9) is semiglobally ISpS (Input-to-State practically Stable) in the sense of Jiang et al. (1994).

Our proof, provided in Section 7.1, is constructive and the comparison functions involved in (10) are given explicitly (cf. (25)). Due to the particular design of the encoding-decoding procedure, measurement errors no longer appear as an input. Indeed, their effects are embedded in the last term of (10), which depends only on the parameters of the digital controller. As already anticipated in Section 4, the constant $\Lambda$ is a measure of the expansion of the system between two successive sampling times. More precisely, as shown in the proof of Theorem $5.1, \Lambda=e^{\mathcal{L} \tau}$, where $\mathcal{L}$ is the Lipschitz constant of $f$ over the region where, by construction, the solutions are guaranteed to lie. On the other hand, the constants $\bar{E}$ and $E$ are proportional to the upper bound on the size of disturbances-uncertainties and measurement errors, respectively, as defined in (3) and (20). Hence, the last term in (10), which constitutes an upper bound to the steady-state error, is a continuous function of the known upper bound on the size of exogenous disturbances, that vanishes at zero. This guarantees that the steady-state error is small if disturbances are small, provided a sufficient knowledge of the plant. Moreover, when no perturbations apply, we recover the exact same result as Liberzon and Hespanha (2005). It is interesting to note that the required data-rate to guarantee this robustness, expressed by relation (14), is the same as in Liberzon and Hespanha (2005).

Robustness to model uncertainties is the main contribution of this work if compared to the existing representative examples in the literature (Kameneva and Nešić (2008) and Sharon and Liberzon (2007)). In Kameneva and Nešić (2008) this lack of robustness is due to the digital nature of the controller, which is based on the exact dynamics or at most on its discrete time approximation (cf. equation (2) and the text below in that reference). In Sharon and Liberzon (2007) this possible lack of robustness comes from the fact that ISS of the quantized closed-loop system is achieved through a cascade reasoning from the quantization error (which is ISS with respect to external disturbances thanks to the particular encoding/decoding strategy) to the system's state (which is ISS by hypothesis). This is possible because the evolution of the quantization error is shown to be independent from both the controller's and the system's state (cf. equation (12) in that reference). This is no longer achievable if one introduces if one introduces parametric uncertainties, as the state of the controller is fed back in the evolution equation of the quantization error.

Another contribution compared to Sharon and Liberzon (2007) is the non-local characterization of robustness, which turns out to be semiglobal. In the statement of Theorem 2 in that reference, which gives an extension of the proposed algorithm to nonlinear systems, the admissible ball of initial conditions and external disturbances are built starting from the $\mathcal{K}_{\infty}$ functions $\bar{\beta}_{c l}$ and $\bar{\gamma}_{c l}$, whose explicit expression depends on the Lipschitz constant of the system (cf. proof of Theorem 1 in that reference). Indeed, given a region on which define the Lipschitz constant is defined $\left(|x|<l_{x}\right.$ and $\left.|w|<l_{w}\right)$, it is possible to find the size of the ball of admissible initial conditions $\delta$ and allowed disturbances $\epsilon$ by satisfying the two relations $\bar{\beta}_{c l}(\delta)+\bar{\gamma}(\epsilon)<l_{x}$ and $\epsilon<l_{w}$. It follows that the value of $\delta$ and $\epsilon$ cannot be chosen a priori, and may result to be impossible to be arbitrarily enlarged, depending on the explicit expression of the two $\mathcal{K}_{\infty}$ functions $\bar{\beta}_{c l}$ and $\bar{\gamma}_{c l}$. On the other hand, given a ball of initial conditions and a bound on the size of exogenous disturbances, it is not possible either to build the $\mathcal{K}_{\infty}$ functions used in the statement of the theorem, as it is not possible to build an "overshoot" region in which the Lipschitz constant would be defined. These observations show that in Sharon and Liberzon (2007) the extension to 
nonlinear systems is only local. In this paper we give a constructive way to build the overshoot region starting from an arbitrary ball of initial conditions and an arbitrary size for the exogenous disturbances.

However, considered the superior performances in the steady-state error of the algorithm proposed in Sharon and Liberzon (2007), that achieves ISS instead of ISpS, one may think of implementing some switching strategy between the two methods. In a first step one would catch the state with the algorithm proposed here even in the case of parametric uncertainties. In a second time, once the parameters of the systems have been identified and the state has entered a sufficiently small region around the origin, one would switch to the one proposed in Sharon and Liberzon (2007).

In case of overflow, the size of quantization region is set to $\Lambda(\bar{E}+E)+E$ (cf. (7)), It may happen, in particular for large sampling period, that $\Lambda \gg 1$. In this case, the quantization error may become very large as $\bar{E}$ depends linearly on $\Lambda$ (cf. (20)), leading to a drop in performances. This can be easily avoided by using, even when no measurement errors are considered, a strictly positive $E$ in the encoding-decoding procedure. Indeed it follows from Claim 7.2 that, as long as no overflow occurs, the size of the quantization region converges to

$$
\mathcal{Q}_{\infty}:=\sigma_{\infty} E,
$$

where $\sigma_{\infty}$ is defined in (22). It follows that the maximum quantization error (6) converges from above to

$$
\bar{e}_{\infty}=\left(\frac{\sigma_{\infty}}{N}+1\right) E .
$$

From (18) it follows that an overflow can occur only if the maximum quantization error gets smaller than $\bar{\eta}$, defined in (15). Hence it suffices to set $E$ such that

$$
\bar{e}_{\infty} \geq \bar{\eta}
$$

to avoid overflows. We then have the following theorem, whose proof follows directly from that of Theorem 5.1, together with Equations (11) and (12).

Theorem 5.2: Under the same assumption of Theorem 5.1, the design parameters $\tau, \Lambda, \bar{E}, E$ can be picked is such a way that (10) holds with $\delta=\bar{\gamma}\left(\left(1+\frac{\Lambda+1}{1-\frac{\Lambda}{N}}\right) E\right)$.

We point out that the size of the steady state error $\delta$ defined in Theorem 5.2 can be either larger or smaller than the one obtained in Theorem 5.1, depending on the parameters involved.

We now state a similar result for the case when the sampling time is fixed and we can only adjust the number of transmittable bits. In this context, it appears that, due to the presence of exogenous perturbations, $\tau$ cannot be chosen arbitrarily large, as it happens in the ideal case (cf. Liberzon and Hespanha (2005)). This intuitive fact is detailed in the proof, given in Section 7.4 .

Theorem 5.3: Let Assumption 3.1 hold for the system (1). Then, there exist class $\mathcal{K}_{\infty}$ functions $\bar{\chi}, \bar{\Gamma}, \bar{\gamma}$ and, given any compact sets $\mathcal{P} \subset \mathbb{R}^{p}, \mathcal{D} \subset \mathbb{R}^{d}$ and any $\Delta \in \mathbb{R}_{>0}$, there exists a time $\tau_{\max } \in \mathbb{R}_{>0}$ such that, for all $\tau \in\left(0, \tau_{\max }\right)$, there exist positive constants $N, \Lambda, \bar{E}, E$ and a class $\mathcal{K} \mathcal{L}$ function $\beta$ such that trajectories of the closed-loop system (9), where $\hat{u}(t)$ is the output of the digital controller defined by equations (4)-(8), satisfy (10) for all $x(0) \in B(0, \Delta)$, all $\nu \in \mathcal{P}$, all $\mu: \mathbb{R}_{\geq 0} \rightarrow \mathcal{P}$, all $d: \mathbb{R}_{\geq 0} \rightarrow \mathcal{D}$ and all $d_{e}: \mathbb{R}_{\geq 0} \rightarrow B(0, E)$. That is, (9) is semiglobally ISpS.

The comparison functions involved in the trajectories estimate of this result are the same as for Theorem 5.1.

Remark 3: Theorem 5.1, main delta N - no overflows and 5.3 can be easily generalized to 
Figure 2. Evolution of the norm of the state from $x(0)=[-10,-10]$.

non constant sampling period, provided that the time between two samples does not exceed the value $\tau$ defined in the above statements. $\triangleleft$

\section{Illustrative Example}

We check the application of our strategy on the control of a model of a DC motor with a load modelled as a nonlinear torque. The uncertainty on the load is modeled by unknown time-varying variables $\mu$ and $d_{1}$. Actuator errors are represented by an exogenous disturbance $d_{2}$ :

$$
\begin{aligned}
& \dot{x}_{1}=x_{2}+\mu x_{1}^{3}+d_{1} \\
& \dot{x}_{2}=u+d_{2} .
\end{aligned}
$$

For the needs of the numerical simulations, we have chosen $\mu(t)=1+P \sin (t), d_{1}(t)=D \sin (t)$ and $d_{2}(t)=D \cos (t)$. At each sampling time the measurement available to the encoder is perturbed by the measurement error $d_{e}(t)=E(\sin (t), \cos (t))^{T}$. The system being in strict feedback form, we follow Freeman and Kokotovich (1993) to construct a continuous $\operatorname{ISS}\left(d, d_{p}, d_{e}\right)$ feedback law. We assume that only 2 bytes can be transmitted at each sampling time. Our aim is to stabilize every solution starting in $B(0,10)$, i.e $\Delta=10$, assuming the following values for the perturbation amplitudes, $P=0.5, D=1.0, E=0.1$. Note that this correspond to a $50 \%$ uncertainty on the load parameter. Our control scheme with parameters $\tau=0.1 s, \Lambda=64, \bar{E}=64, E$ and $\nu=1$ successfully stabilizes the system (cf. fig. 2).

Since $E>0$, the size of the quantization region remains sufficiently large and no overflow occurs, as anticipated in Section 5. If an overflow had occurred then the size of the quantization region would have jumped to $\Lambda(\bar{E}+E)+E$ (cf. (7)), leading to a big drop in performances. This illustrates the fact that, even when no measurement error applies, setting an appropriate $E>0$ may be very profitable in practice.

For $\Delta=5$, and the same parameters, the sampling period can be taken as large as $0.3 \mathrm{~s}$. We point out that the good performance of our strategy are also due to the ISS characteristics of the feedback strategy provided in Freeman and Kokotovich (1993).

In conclusion, with only two bytes, with a sampling period of the order of the plant's time scale, and with disturbance magnitude of the order of the nominal controlled dynamics values, our proposed approach succeeds in stabilizing the system, with a steady-state error of the same magnitude as the perturbations.

\section{$7 \quad$ Proof of the main results}

\subsection{Proof of Theorem 5.1}

Contraction of the quantization region: Suppose there are no measurement errors, i.e $E=0$. Then we want the estimation error to decrease as long as no overflow occurs, that is, as long as $x(k \tau) \in \mathcal{Q}(k \tau)$, we impose $L(k \tau)<L((k-1) \tau)$. This means, in view of $(5)$, that

$$
\frac{\Lambda}{N}<1
$$

Divergence between sampling times: During the time intervals separating two consecutive sampling times, the estimation error may increase. To evaluate this expansion, let us assume 
that a number $\hat{W}>0$ is known $^{1}$ such that $x(t)+d_{e} \in B(0, \hat{W})$ and $\hat{x}(t) \in B(0, \hat{W})$ for all $t \geq 0$. In this case, it results from the continuity of $\kappa$ that, for all $t \geq 0,|\hat{u}(t)| \leq$ $\max _{\hat{x} \in B(0, \hat{W}), \nu \in \mathcal{P}}|\kappa(\hat{x}, \nu)|=: U<\infty$. Let $\mathcal{L}(\hat{W})$ be the Lipschitz constant of $f$ over the region $\left\{(x, \mu, u, d) \in \mathbb{R}^{n+p+m+h}:|x| \leq \hat{W}, \mu \in \mathcal{P},|u| \leq U, d \in \mathcal{D}\right\}$, then, in view of (4) and exploiting the Bellman-Gronwell Lemma, it holds that, for all $x, \hat{x} \in B(0, \hat{W}), \frac{d}{d t}|e(t)| \leq$ $|f(x, \mu(t), \hat{u}, d(t))-f(\hat{x}, \nu, \hat{u}, 0)| \leq \mathcal{L}(\hat{W}) \max \{|e(t)|, \eta(t)\}$, where $\eta(t)=\max \{|\mu(t)-\nu|,|d(t)|\}$. Note that $\eta(t) \leq \bar{\eta}$, where

$$
\bar{\eta}=\max \left\{\sup _{\mu^{\prime}, \nu^{\prime} \in \mathcal{P}}\left|\mu^{\prime}-\nu^{\prime}\right|, \sup _{d^{\prime} \in \mathcal{D}}\left|d^{\prime}\right|\right\} .
$$

Hence, it holds that, for all $t \neq k \tau, k \in \mathbb{N}$,

$|e(t)| \geq \eta(t) \quad \Rightarrow \quad \frac{d}{d t}|e(t)|=\mathcal{L}(\hat{W})|e(t)|$, and

$|e(t)|<\eta(t) \quad \Rightarrow \quad \frac{d}{d t}|e(t)|=\mathcal{L}(\hat{W}) \eta(t)$.

By the fact that $|e(0)| e^{\mathcal{L}(\hat{W}) t}<|e(0)|+\bar{\eta} \mathcal{L}(\hat{W}) t$ only if $|e(0)|<\bar{\eta} \frac{\mathcal{L}(\hat{W}) t}{e^{\mathcal{L}(\hat{W}) t}-1} \leq \bar{\eta}$, it follows that, for all $t \in[0, \tau]$,

$$
|e(0)| \geq \bar{\eta} \Rightarrow|e(t)| \leq|e(0)| e^{\mathcal{L}(\hat{W}) t} .
$$

Defining $\Lambda=e^{\mathcal{L}(\hat{W}) \tau}$, we can give a natural interpretation to (5). The constant $N^{-1}$ describes the effect of measuring the state, which individuates a smaller hypercube to which the state belongs, while $\Lambda$ describes the increase in the size of this hypercube during the time before the next sampling to make sure the state belongs to the new quantization region. Recalling that $|e(k \tau)| \leq \bar{e}(k \tau)$ for all $k \in \mathbb{N}$, we claim that

$$
\bar{e}(k \tau) \geq \bar{\eta} \Rightarrow \mid e\left((k+1) \tau^{-} \mid \leq \Lambda \bar{e}(k \tau)\right.
$$

that is $x((k+1) \tau) \in \mathcal{Q}((k+1) \tau)$ and, consequently, $q_{k+1}>0$ (i.e. no overflow at step $\left.k+1\right)$. Indeed, note that if $\bar{\eta} \leq|e(k \tau)| \leq \bar{e}(k \tau)$, then (17) follows from (16), while, if $|e(k \tau)|<\bar{\eta}$, there exists $\tilde{t}:=\min \{t \geq 0:|e(k \tau)|+\bar{\eta} \mathcal{L}(\hat{W}) t \geq \bar{\eta}\}$. If $\tilde{t} \geq \tau$ then $\left|e\left((k+1) \tau^{-}\right)\right|<\bar{\eta}<\Lambda \bar{e}(k \tau)$, while, if $\tilde{t}<\tau$, then, by (16), $\left|e\left((k+1) \tau^{-}\right)\right| \leq \bar{\eta} e^{(\tau-\tilde{t}) \mathcal{L}(\hat{W})}<\Lambda \bar{\eta} \leq \Lambda \bar{e}(k \tau)$, which shows (17).

Furthermore, by reversing (17), we obtain that $\mid e\left((k+1) \tau^{-} \mid>\Lambda \bar{e}(k \tau)\right.$ only if $\bar{e}(k \tau)<\bar{\eta}$, that is, defining $E=\sup _{d_{e}^{\prime} \in \mathcal{E}}\left|d_{e}\right|, \mid e\left((k+1) \tau^{-} \mid+E>L((k+1) \tau)\right.$ only if $\bar{e}(k \tau)<\bar{\eta}$, which implies that an overflow may occur only when the maximum quantization error (6) is below the bound $\bar{\eta}$ :

$$
q_{k+1}=0 \quad \Rightarrow \quad \bar{e}(k \tau)<\bar{\eta}
$$

Moreover, we establish the following upper bound on the size of the estimation error right before an overflow:

$$
q_{k+1}=0 \quad \Rightarrow \quad\left|e\left((k+1) \tau^{-}\right)\right|<\Lambda \bar{\eta} .
$$

Note that, from (18), there exists a time $\tilde{t}^{\prime}:=\min \{t>0:|e(k \tau)|+\bar{\eta} \mathcal{L}(\hat{W}) t \geq \bar{\eta}\}$. If $\tilde{t}^{\prime} \geq \tau$ then $\left|e\left((k+1) \tau^{-}\right)\right| \leq \bar{\eta}<\Lambda \bar{\eta}$. On the other hand, if $\tilde{t}^{\prime}<\tau$ then, by $(16),\left|e\left((k+1) \tau^{-}\right)\right| \leq$ $\bar{\eta} e^{\left(\tau-\tilde{t}^{\prime}\right) \mathcal{L}(\hat{W})}<\Lambda \bar{\eta}$, which establishes (19).

\footnotetext{
${ }^{1}$ We will demonstrate the existence of such $\hat{W}$, by constructing it, in the sequel (cf. Claim 7.1 ).
} 
Trajectory boundness: Fix any $\Lambda>1$ and let

$$
\bar{E}=\Lambda \bar{\eta} .
$$

By equation (19) and as long as $x(t)+d_{e} \in B(0, \hat{W})$, this implies that, in the eventuality of an overflow at time $k_{0} \tau$ (i.e. $q_{k_{0}}=0$ ),

$$
x\left(k_{0} \tau\right), x\left(k_{0} \tau\right)+d_{e} \in B\left(\hat{x}\left(k_{0} \tau\right), \bar{E}+E\right),
$$

In view of (18) and $(7)$, this implies that $x\left(\left(k_{0}+1\right) \tau\right) \in \mathcal{Q}\left(\left(k_{0}+1\right) \tau\right)$, that is $q_{k_{0}+1}>0$. This means that it is not possible to have two successive overflows.

Let $\Omega_{c}:=\left\{x \in \mathbb{R}^{n}: V(x) \leq c\right\}$, where

$c=\bar{\alpha}\left(\chi\left(\sup _{d^{\prime} \in \mathcal{D}}\left|d^{\prime}\right|\right)+\Gamma\left(\sup _{\mu^{\prime}, \nu^{\prime} \in \mathcal{P}}\left|\mu^{\prime}-\nu^{\prime}\right|\right)+\gamma\left(\max \left\{\Delta+E+\sigma_{\infty} E, \Lambda(\bar{E}+E)+E\right\}\right)\right)$, where $\sigma_{\infty}$ is defined as

$$
\sigma_{\infty}:=(\Lambda+1) N /(N-\Lambda)
$$

Let

$$
\begin{gathered}
\hat{W}:=W+\max \left\{\Delta+E+\sigma_{\infty} E, \Lambda(\bar{E}+E)+E\right\}, \\
W:=\max \left\{\Delta, \sup _{x, z \in \Omega_{c}}|x-z|\right\},
\end{gathered}
$$

and pick the sampling period $\tau$ as $\tau=\frac{\ln (\Lambda)}{\mathcal{L}(\hat{W})}$. We prove the following in Sections 7.2 and 7.3 respectively.

Claim 7.1 The solutions of the closed-loop system satisfy $|x(t)| \leq W, \quad|\hat{x}(t)| \leq \hat{W}, \quad \forall t \geq 0$.

Claim 7.2 As long as no overflow occurs, it holds that $|e(t)| \leq\left(\frac{\Lambda}{N}\right)^{t / \tau} L(0)+\sigma_{\infty} E$.

Conclusion: From the proof Claim 7.1, it results that, for all $t \geq 0,|e(t)| \leq \max \left\{(\Delta+E) e^{-\lambda t}+\right.$ $\left.\sigma_{\infty} E, \Lambda(\bar{E}+E)+E\right\} \leq \Delta e^{-\lambda t}+\Lambda(\bar{E}+E)+\left(2+\sigma_{\infty}\right) E, \forall t \geq 0$. From Assumption 3.1, this implies that the trajectories of the closed loop system (9), with parameters $\{N, \tau, \Lambda, \bar{E}, \nu, E\}$, satisfy

$$
|x(t)| \leq \underline{\alpha}^{-1}\left(\bar{\alpha}\left(\gamma\left(\Delta e^{-\lambda t}\right)+\chi(\|d\|)+\Gamma(\|\mu-\nu\|)+\gamma\left(\Lambda(\bar{E}+E)+\left(2+\sigma_{\infty}\right) E\right)\right)\right),
$$

for all $x(0) \in B(0, \Delta)$, all $\nu \in \mathcal{P}$, all $\mu: \mathbb{R}_{\geq 0} \rightarrow \mathcal{P}$, all $d: \mathbb{R}_{\geq 0} \rightarrow \mathcal{D}$ and all $d_{e}: \mathbb{R}_{\geq 0} \rightarrow B(0, E)$. From this and from the fact that $\sigma(a+b) \leq \sigma(2 a)+\sigma(2 b)$ for all nondecreasing function $\sigma$ and all $a, b \geq 0$, the theorem is proved with

$$
\begin{aligned}
\bar{\beta}(\cdot, t) & =\underline{\alpha}^{-1}\left(\bar{\alpha}\left(2 \gamma(\cdot) \gamma\left(e^{-\lambda t}\right)\right)\right) \\
\bar{\chi}(\cdot) & =\underline{\alpha}^{-1}(\bar{\alpha}(4 \chi(\cdot))) \\
\bar{\Gamma}(\cdot) & =\underline{\alpha}^{-1}(\bar{\alpha}(8 \Gamma(\cdot))) \\
\bar{\gamma}(\cdot) & =\underline{\alpha}^{-1}(\bar{\alpha}(8 \gamma(\cdot))) .
\end{aligned}
$$

\subsection{Proof of Claim 7.1}

Let $\Theta:=\inf \left\{t \in \mathbb{R}_{>0}:|x(t)|>W\right.$ or $\left.|\hat{x}(t)|>\hat{W}\right\}$. This time is well defined as $|x(0)| \leq$ $\Delta \leq W$ and, from the fact that $q_{0}>0$ by construction, $\hat{x}(0) \in B(0, \Delta+E) \subset B(0, \hat{W})$. For all 
$t \in[0, \Theta), \mathcal{L}(\hat{W})$ can be correctly interprated as an upper bound on the expansion of the system. In particular, Claim 7.2, (18) and (19) hold for all $t \in[0, \Theta)$.

Define $k_{0} \tau$ as the time of the first overflow. Then, by Claim 7.2, it holds that, for all $t \in$ $\left[0, \min \left(\Theta,\left(k_{0}-1\right) \tau\right)\right),|e(t)|<L(0) e^{-\lambda t}+\sigma_{\infty} E \leq \Delta+E+\sigma_{\infty} E$, where $\lambda:=\frac{\left|\ln \left(\frac{\Lambda}{N}\right)\right|}{\tau}$.

If $\Theta<\left(k_{0}-1\right) \tau$, then, from Assumption 3.1 and (A.Isidori 1999, Section 10.4), it results that the set $\Omega_{\tilde{c}}=\left\{x \in \mathbb{R}^{n}: V(x) \leq \tilde{c}\right\}$, where $\tilde{c}:=\bar{\alpha}\left(\chi\left(\sup _{d^{\prime} \in \mathcal{D}}\left|d^{\prime}\right|\right)+\Gamma\left(\sup _{\mu^{\prime}, \nu^{\prime} \in \mathcal{P}}\left|\mu^{\prime}-\nu^{\prime}\right|\right)+\gamma(\Delta+\right.$ $\left.E+\sigma_{\infty} E\right)$ ), is an invariant attractive set, and, noting that $\tilde{c} \leq c$, it follows that $\Omega_{\tilde{c}} \subseteq \Omega_{c}$, which, by the definition of $W$ in (24), implies $|x(t)| \leq W$ for all $t \in[0, \Theta]$. This in turn ensures that $\sup _{t \in[0, \Theta]}|\hat{x}(t)| \leq W+\sup _{t \in[0, \Theta]}|e(t)| \leq W+\Delta+E+\sigma_{\infty} E \leq \hat{W}$ (cf. (23)). This contradicts the definition of $\Theta$ and hence we conclude that $\Theta \geq\left(k_{0}-1\right) \tau$.

If $\Theta \in\left[\left(k_{0}-1\right) \tau, k_{0} \tau\right)$, then, by (18) and (19) and the definition of $\bar{E}$ in (20), it results that $|e(t)|<\bar{E}$ for all $t \in\left[t_{0}-\tau, \Theta\right]$. With the same arguments as before, this contradicts the definition of $\Theta$ and hence we conclude $\Theta \geq k_{0} \tau$.

If $\Theta \in\left[k_{0} \tau,\left(k_{0}+1\right) \tau\right)$, by $\bar{E}>\bar{\eta}$ and (21), we get that $|e(t)| \leq \Lambda(\bar{E}+E)$ for all $t \in\left[k_{0} \tau, \Theta\right]$, again contradicting the definition of $\Theta$. Hence we can conclude $\Theta \geq\left(k_{0}+1\right) \tau$.

If $\Theta=\left(k_{0}+1\right) \tau$, then by construction $q_{k_{0}+1}>0$ and $\left|e\left(\left(k_{0}+1\right) \tau\right)\right| \leq L\left(\left(k_{0}+1\right) \tau\right)=$ $\Lambda(\bar{E}+E)+E$, again contradicting the definition of $\Theta$. Hence $\Theta>\left(k_{0}+1\right) \tau$.

The system properties established along the whole proof being uniform in time, we can set $t^{\prime}=t-\left(k_{0}+1\right) \tau$ and apply the same arguments with new "initial" condition $L(0)=\Lambda(\bar{E}+E)+E$ until the next overflow. By reiterating for successive overflows, we conclude $\Theta=\infty$, which is enough to prove the claim.

\subsection{Proof of Claim 7.2}

The first line in (5), can be rewritten as

$$
L(k \tau)=R^{k} L(0)+E(\Lambda+1) \sum_{i=0}^{k} R^{i}<\tilde{L}(k \tau)
$$

where $\tilde{L}: \mathbb{R}_{\geq 0} \rightarrow \mathbb{R}_{>0}$ is defined as

$$
\tilde{L}(t)=R^{t / \tau} L(0)+E(\Lambda+1) \sum_{i=0}^{\infty} R^{i}
$$

and $R:=\Lambda / N<1$ (cf. (14)).

If $\Lambda$ is chosen appropriately to compensate for error divergences between sampling times, if no overflow occurs, then, recalling the definition of the maximum quantization error (6), forall $t \in[k \tau,(k+1) \tau),|e(t)| \leq \Lambda^{\frac{t-k \tau}{\tau}}\left(\frac{L(k \tau)}{N}+E\right)$. Substituting (26) in the this equation, we obtain that, for all $t \in[k \tau,(k+1) \tau)$,

$$
|e(t)| \leq\left(\frac{\Lambda}{N}\right)^{t / \tau} L(0)+\frac{\Lambda^{\frac{t-k \tau}{\tau}}}{N} E(\Lambda+1) \sum_{i=0}^{k}\left(\frac{\Lambda}{N}\right)^{i}+\Lambda E<\tilde{L}(t) .
$$

Recalling finally that the geometric series in the definition of $\tilde{L}$ in $(27)$ converges, $\sum_{i=0}^{\infty}\left(\frac{\Lambda}{N}\right)^{i}=$ $\frac{N}{N-\Lambda}$, this finishes to establish Claim 7.2 by recalling the definition of $\sigma_{\infty}$ in (22) and by noticing that the error can only decrease at the sampling times. 


\subsection{Proof of Theorem 5.3}

The proof follows along the same lines as that of Theorem 5.1. The difference stands in the fact that $\Lambda$ can no longer be chosen arbitrarily. Instead, given any $\tau>0$, we define $\Lambda=e^{\mathcal{L}(\hat{W}) \tau}$, where $\hat{W}$ is defined as before (cf. (23)). Note that $\Lambda$ enters the definition of $\hat{W}$, in particular $\hat{W}$ and $\mathcal{L}(\hat{W})$ are both continuous increasing functions of $\Lambda$. Hence, $\Lambda$ is required to satisfy the following two equations $\Lambda=e^{\mathcal{L}(\hat{W}) \tau}$ and $\hat{W}=\hat{W}(\Lambda)$. This set of equations admits other solutions than the trivial one $(\Lambda=1, \tau=0)$ provided that

$$
\left.\tau e^{\mathcal{L}(\hat{W}(\Lambda)) \tau} \frac{d}{d \Lambda} \mathcal{L}(\hat{W}(\Lambda))\right|_{\Lambda=1}<1
$$

By continuity of the equations in $\tau$ and $\Lambda$, we conclude that there exists $\tau_{\max }>0$ such that a solution exists for all $\tau \in\left(0, \tau_{\max }\right)$. The rest of the proof follows that of Theorem 5.1.

\section{Conclusion}

The proposed strategy for limited-information feedback control of nonlinear plants is shown to be robust to exogenous disturbances and measurement errors, even in presence of parametric uncertainty. Its application is illustrated by the numerical simulation of a DC motor control. Possible future extensions concern output feedback (see Sharon and Liberzon (2008) for a representative example) and robustness to delays.

\section{References}

A.Isidori,, Nonlinear control system II, Springer Verlag (1999).

Freeman, R.A., and Kokotovich, P.V. (1993), "Global Robustness of Nonlinear Systems to State Measurment Disturbances," IEEE 32nd Conference on Decision and Control, pp. 15071512 .

Hespanha, J.P., Naghshtabrizi, P., and Xu, Y. (2007), "A SURVEY OF RECENT RESULTS IN NETWORKED CONTROL SYSTEMS," Proceedings of the IEEE, 95, 138-162.

Jaglin, J., de Wit, C.C., and Siclet, C. (2008), "Delta Modulation for Multivariable Centralized Linear Networked Controlled Systems," in .

Jaglin, J., de Wit, C.C., and Siclet, C. (2009), "Adaptive quantization for linear systems," Submitted to IEEE Conf. on Decision and Control 2009.

Jiang, Z.P., Teel, A., and Praly, L. (1994), "Small gain theorems for ISS systems and applications," Math. of Cont. Sign. and Syst., 7, 95-120.

Kameneva, T., and Nešić, D. (2008), "Input-to-State Stabilization of Nonlinear Systems with Quantized Feedback," in Proc. 17th. IFAC World Congress, pp. 12480-12485.

Liberzon, D. (2003), "On stabilization of linear systems with limited information," IEEE Trans. on Automat. Contr., 48, 304-307.

Liberzon, D. (2008), "Nonlinear control with limited information," Submitted to Communications in Information Systems, Roger Brockett Legacy special issue. Available at http://decision.csl.uiuc.edu/ liberzon/research/legacy.pdf.

Liberzon, D., and Hespanha, J.P. (2005), "Stabilization of Nonlinear Systems With Limited Information Feedback," IEEE Trans. on Automat. Contr., 50, 910-915.

Liberzon, D., and Nešić, D. (2007), "Input-to-state stabilization of linear systems with quantized state measurements," IEEE Trans. on Automatic Control, 52, 767-781.

Montestruque, L.A., and Antsaklis, P. (2004), "Stability of model-based networked control systems with time-varying transmission times," IEEE Trans. on Automat. Contr., 49, 15621572 . 
Nair, G.N., and Evans, R.J. (2004), "Stabilizability of stochastic linear systems with finite feedback data rates," SIAM J. Control Optim., 43, 413-436.

Nair, G.N., Fagnani, F., Zampieri, S., and Evans, R.J. (2007), "Feedback Control Under Data Rate Constraints: An Overview," Proc. of the IEEE, 95, 108-137.

Persis, C.D., and Isidori, A. (2004), "Stabilizability by State Feedback Implies Stabilizability by Encoded State Feedback," System \& Control Letters, 53, 249-258.

Petersen, I.R., and Savkin, A.V. (2001), "Multi-rate stabilization of multivariable discrete-time linear systems via a limited capacity communication channel," Proc. 40th IEEE Conf. on Decision and Control.

Sharon, Y., and Liberzon, D. (2007), "Input-to-state stabilization with minimum number of quantization regions," in , pp. 20-25.

Sharon, Y., and Liberzon, D. (2008), "Input-to-state stabilization with quantized output feedback," in , pp. 500-513.

Sontag, E., and Wang, Y. (1995), "On characterizations of the input-to-state stability property," Syst. \& Contr. Letters, 24, 351-359. 